\title{
PERFIL EPIDEMIOLÓGICO DAS LESÕES MUSCULOESQUELÉTICAS OBSERVADAS NA CLÍNICA DA FACULDADE SANTA MARIA
}

\author{
Levi Felipe Gomes ${ }^{1}$ \\ Emanuely Rolim Nogueira ${ }^{2}$ \\ Michel Jorge Dias ${ }^{3}$ \\ Juliane Carla Medeiros de Sousa ${ }^{4}$
}

RESUMO: Objetivo: Traçar perfil epidemiológico das lesões musculoesqueléticas observadas na clínica da Faculdade Santa Maria. Método: Trata-se de uma pesquisa exploratória, descritiva, transversal, retrospectiva, com base em dados secundários proveniente de prontuários a ser realizada na clínica da Santa Maria em Cajazeiras-PB. Utilizou-se de questionários para captação de dados clínicos e epidemiológicos de 200 prontuários disponíveis dos últimos 5 anos. Foram excluídos aqueles que não assinaram o Termo de Consentimento no ato da admissão do paciente. As análises estatísticas foram realizadas com auxílio do software SPSS (Statistical Package for the Social Sciences) versão 20.0. Resultados: Evidenciouse uma prevalência do sexo feminino e adultos jovens em $49 \%$ da amostra, $20 \%$ de agricultores, com $47 \%$ de disfunções ortopédicas de acometimento da coluna vertebral. As algias foram as queixas mais relevantes e a patologia associada mais presente foi a hipertensão arterial sistêmica; justificando os altos índice de uso de anti-hipertensivos. No tratamento o enfoque para recursos de cinesioterapia realizados em $100 \%$ da amostra. Considerações finais: Os resultados obtidos mostram índices elevados do perfil epidemiológico de disfunções musculoesqueléticas. Evidencia-se a necessidade de uma contínua avaliação dos prontuários, visando minimizar incidências patológicas do sistema musculoesquelético para os pacientes que procuram os serviços de Fisioterapia.

Palavras chave: Epidemiologia. Lesões musculoesqueléticas. Perfil.

\footnotetext{
${ }^{1}$ Acadêmico de Fisioterapia da Faculdade Santa Maria. Cajazeiras-PB.

${ }^{2}$ Fisioterapeuta. Especialista em Recursos Cinesioterapêuticos pela Faculdade Santa Maria. Docente da Faculdade Santa Maria, Cajazeiras, PB.

${ }^{3}$ Fisioterapeuta. Mestre em Saúde Coletiva pelo Programa Stricto Sensu da Universidade Católica de Santos. Santos, SP, Brasil. Docente da faculdade Santa Maria, Cajazeiras, PB.

${ }^{4}$ Fisioterapeuta. Mestre em Saúde Coletiva pelo Programa Stricto Sensu da Universidade Católica de Santos. Santos, SP, Brasil. Docente da faculdade Santa Maria, Cajazeiras, PB.
} 


\section{INTRODUÇÃO}

As disfunções musculoesqueléticas são compreendidas como as doenças do sistema locomotor e também do tecido conectivo, sendo considerada a causa mais comum de incapacidade crônica no mundo (DAVID, 2017).

Afetam cerca de 60 milhões de pessoas mundialmente, tornando-se um fator contribuinte no alto índice de internações hospitalares. Conforme dados da Organização Mundial de Saúde (OMS) o número de óbitos por traumatismos chega a aproximadamente 8,5 milhões na população mundial (SANTOS et al., 2016; PEREIRA et al., 2013).

As lesões ocupam o primeiro lugar das etiologias de morbimortalidade entre 0 a 39 anos de idade, sendo responsável por 150.000 mortes por ano, as quais podem ser acarretadas por fatores genéticos, biológicos, psicossociais ou relacionados à idade. Os comprometimentos mais frequentes são as patologias da coluna vertebral, seguidas das disfunções de ombros, cotovelos, punhos, mãos e membros inferiores de origem traumática/ortopédica (DAVID, 2017; NOGUEIRA et al., 2016; WATANABE, 2012).

Dentre as doenças degenerativas mais comuns observamos a Osteoatrose, Artrite Reumatóide, Osteoporose e a Osteopenia, que como consequência ocasionam perda da funcionalidade, dores, inflamações, alterações estruturais, desequilíbrios fisiológicos, deformidades e limitações das atividades de vida diárias (SANTOS et al., 2016).

Nos serviços de saúde, é comum a prevalência de disfunções como fraturas, rigidez articular e hérnias discais, estiramento muscular, tendinite, rupturas tendinosas, distensões e desinserção tendinosas e lesões de ligamentos (SILVA et al., 2015, SOUSA et al., 2011). O meio essencial para tratamento de tais lesões é a Fisioterapia, que contribui na prevenção, promoção de saúde e reabilitação dos distúrbios que acometem a funcionalidade corporal, permitindo o retorno breve e independência das atividades de vida diária (OLIVEIRA et al., 2018; OLIVEIRA; BRAGA 2010). 
Observando que as disfunções musculoesqueléticas são as principais causas de complicações do sistema locomotor e que essas trazem prejuízo a vida das pessoas, geram impactos significativos na sua funcionalidade e qualidade de vida. Com isso, é importante os estudos epidemiológicos por proporcionar a compreensão dos fenômenos que englobam a saúde da população, e através do desfecho salientar a contribuição da fisioterapia na prevenção, promoção e recuperação da saúde.

O objetivo desse estudo foi traçar perfil epidemiológico das lesões musculoesqueléticas observadas na Clínica da Faculdade Santa Maria.

\section{MÉTODO}

Trata-se de uma pesquisa exploratória, descritiva, transversal, retrospectiva, com base em dados secundários proveniente dos prontuários de pacientes com lesões musculoesqueléticas atendidos no setor de Fisioterapia de traumatoortopedia da Faculdade Santa Maria, sendo realizada entre os meses de março e abril de 2019.

Foram avaliados 200 prontuários, onde os critérios de inclusão contemplaram prontuários disponíveis dos últimos 5 anos de pacientes assistidos com disfunções musculo esqueléticas. Foram excluídos aqueles que não assinaram o Termo de Consentimento no ato da admissão do paciente ao serviço.

Para a realização da coleta de dados, foi utilizado um questionário elaborado pelos pesquisadores, contendo as seguintes variáveis: dados demográficos, características clínicas e conduta fisioterapêutica, o qual ocorreu nas dependências da Clínica Santa Maria, sob supervisão do responsável pelo setor em dias aleatórios de acordo com sua disponibilidade. Após a coleta foi submetido a análises estatísticas com auxílio do software SPSS (Statistical Package for the Social Sciences) versão 20.0. As variáveis qualitativas estão apresentadas em termos de seus valores absolutos e relativos; e as variáveis quantitativas em termos de seus 
valores de tendência central e de dispersão. Em seguida os dados foram confrontados com a literatura pertinente.

A pesquisa foi apreciada e aprovada pelo Comitê de Ética da Faculdade Santa Maria - FSM, sob número do parecer 3.258.208 e CAAE 11125319.5.0000.5180 solicitado que o responsável pela Clínica da Faculdade Santa Maria assine o Termo de Consentimento para uso de dados (BRASIL, 2012).

\section{RESULTADOS}

Foram selecionados 200 prontuários de pacientes com disfunções ortopédicas, traumatológicas e reumáticas de ambos os sexos, sendo 130 mulheres e 70 homens, com média de idade de 44,1 \pm 9,5 com índice maior de acometimento no sexo feminino, entre adultos jovens totalizando um percentual de $49 \%$.

Em ambos os sexos é possível observar uma grande variedade de ocupações, bem como de indivíduos que declararam não ter ocupação. Ressaltando uma porcentagem maior na ocupação entre agricultores, para ambos os sexos (Feminino 36, Masculino 10) perfazendo $20 \%$ do total da amostra.

Conforme a classificação por área seja de causa traumatologia, reumatologia e ortopedia; observou-se de forma geral que as de causas ortopédicas foram responsáveis pelo maior número de casos atendidos no universo estudado. $\mathrm{Na}$ variável, as mulheres foram mais acometidas pelas disfunções ortopédicas; sendo que, nos homens as disfunções traumáticas estiveram mais presentes, ambos permeando mais de $45 \%$ da amostra.

Em relação ao acometimento das regiões topográficas, de acordo com cada área clínica, a coluna foi o seguimento mais predominantemente acometido entre a amostra com disfunções ortopédica, representando $42 \%$ dos casos, os membros inferiores foram alvo das principais lesões em traumatologia e reumatologia.

Considerando o acometimento a partir do sexo, verificou-se uma homogeneidade entre a amostra, visto que ambos possuem maior representativa de afecções na coluna vertebral, com valores de $29 \%$ e $17,5 \%$ respectivamente entre 
mulheres e homens. As principais queixas, as algias foram relatadas em grande parte da amostra alcançando $80 \%$ para ambos os sexos em todas as faixas etárias.

Em relação aos exames de imagem realizados, os métodos de Radiografia Convencional, Tomografia Computadorizada, Ressonância Magnética e Ultrassonografias foram encontrados na amostra. Ressalta-se que em alguns casos mais de um tipo de exame era identificado em um mesmo paciente, contrapondo outros que não apresentaram nenhum exame complementar. Nessa perspectiva observa-se que os exames de maior realização foram a radiografia convencional, efetuado por $45,5 \%$ da amostra; assim como a Tomografia Computadorizada encontrada em $27 \%$ dos casos.

De acordo com os prontuários analisados $51 \%$ dos indivíduos faziam uso de alguma medicação, atingindo a margem de $10 \%$ para anti-hipertensivos e valor similar para anti-inflamatórios, seguidos pelo uso de analgésicos.

Em observância as patologias associadas as disfunções ortopédicas, traumáticas e reumáticas, verificou-se que diabetes mellitus, osteoporose, câncer, cardiopatias e hipertensão arterial estiveram presentes na amostra estudada, sendo esta última a de maior apresentação em ambos os sexos, presente em $21,5 \%$ dos casos.

O diagnóstico cinético-funcional apresentou grande variabilidade não sendo possível estabelecer valores numéricos, no entanto, houve similaridade em comprometimentos da mobilidade, desempenho muscular, paresia, equilíbrio e marcha. A média de tempo de atendimento fisioterapêutico foi de $2,3 \pm 1,3$.

Foi possível descrever os recursos fisioterapêuticos utilizados durante os tratamentos, distribuídos entre eletrotermofototerapia, com ênfase para o TENS (Estimulação Elétrica Nervosa Transcutânea); Recursos Terapêuticos Manuais, onde as mobilizações articulares tiveram destaque; Cinesioterapia, com os alongamentos presentes em todos os protocolos fisioterapêuticos e hidrocinesioterapia, sendo utilizada em $13 \%$ da amostra. 


\section{DISCUSSÃO}

Em nosso estudo, após traçar perfil epidemiológico das disfunções musculoesqueléticas de 200 pacientes, foi evidenciado maior prevalência em adultos jovens de todas as faixas etárias, gênero feminino e em agricultores.

No estudo de Levoratto et al. (2014) os adultos jovens com faixa etária compreendida entre 18 e 49 anos possuíram baixa procura por serviço de saúde, o que os autores consideraram um fator de risco para esse grupo populacional; o que difere dos achados no presente estudo, assim como Silva et al. (2015) que encontrou resultados contraditórios ao nosso quanto a análise de 274 prontuários, no qual $55 \%$ dos pacientes eram do sexo masculino.

Em relação a ocupação, nos achados da pesquisa de Jorge e Ambrosio (2016) não constavam índices de agricultores, a maioria dos pacientes investigados $(n=347,48 \%)$ apresentam ensino superior completo, seguido por estudantes ( $n=$ $112,15,5 \%)$ e aposentados ( $n=110,15,2 \%)$.

No presente estudo foi possível observar que as disfunções ortopédicas tem maior incidência referente a coluna vertebral, com índice de $42 \%$ dos casos. Em relação às disfunções traumáticas e reumáticas os membros inferiores foram as regiões mais acometidas. Contudo, David (2017), em análise de 174 prontuários, mostrou que as regiões mais acometidas foram de $19,5 \%$ no joelho, seguido pela região do ombro em $14 \%$, coluna lombar em apenas $12 \%$ dos casos.

Verificou-se que o índice maior de acometimento de disfunções ortopédicas, traumáticas e reumáticas esteve mais presente no sexo feminino e a região mais acometida foi a coluna vertebral com $29 \%$ dos casos. Corroborando, Cerdeira et al (2013) em investigação realizada na clínica escola de fisioterapia no sertão central do Ceará também encontrou a lesão de coluna predominante em mulheres como maior representatividade do seu estudo, totalizando 79 (32,5\%) dos prontuários.

$\mathrm{Na}$ análise relacionada à queixa principal obteve uma maior predominância nas algias com $n=160(80 \%)$ dos casos, dor irradiada $n=17(8,5 \%)$, paresia $N=10$ $(5 \%)$, parestesia $\mathrm{N}=6(3 \%), \mathrm{ADM}$ diminuída $\mathrm{N}=3(1,5 \%)$, bem como mostra Moraes 
(2016) em seu estudo ao relatar que 67,9\% dos pacientes que chegam à clínica escola possuem a dor como queixa principal.

A avaliação da realização de exames de imagens, houve uma maior prevalência nos exames de raio-x $(\mathrm{N}=91)$, assim como também de Tomografia Computadorizada ( $\mathrm{N}=54)$. Sabe-se que a radiografia consta de um recurso de baixo custo e fácil acesso a população tornando-o mais presente entre os exames complementares (SACON, 2011) que em sua pesquisa obteve resultados equivalentes aos apresentados nesse estudo, o qual os exames de raio $\mathrm{x}$ obtiveram maior índice.

$\mathrm{Na}$ análise das patologias associadas e relacionada ao sexo, obtivemos o número de 88 pacientes que apresentaram essa condição associada, representando $21,5 \%$ dos casos em ambos os sexos. O que tem relação com os medicamentos em uso que em sua grande maioria são anti-hipertensivos (10\%) e anti-inflamatórios (10\%), assim como Rios (2009) também mostra em sua pesquisa, que em 41,8\% prontuários de pacientes com queixas musculoesqueléticas, apresentaram associação positiva com a hipertensão arterial sistêmica $(p=0,010)$.

Referente aos recursos terapêuticos os mais utilizados durante o tratamento a Eletrotermofototerapia, Recursos Terapêuticos Manuais, Cinesioterapia e Hidrocinesioterapia estiveram presentes nas condutas; onde a Cinesioterapia contemplou 100\% da amostra. David et al. (2017) também apontam a modalidade cinesioterapia como de grande abordagem através de recursos mecânicos e manuais.

\section{CONSIDERAÇÕES FINAIS}

Ao analisar o perfil epidemiológico das lesões musculoesqueléticas observadas na clínica da Santa Maria, pode-se destacar a presença do sexo feminino, adultos jovens, agricultores, com condições em sua grande maioria ortopédica, de acometimento da coluna vertebral. As algias foram as queixas mais relevantes e a patologia associada mais presente foi a hipertensão arterial sistêmica, 
o que justifica os altos índice de uso de anti-hipertensivos, além de antiinflamatórios. Em relação aos recursos fisioterapêuticos, a cinesioterapia e a eletroterapia são os mais utilizados. Os resultados obtidos são condizentes com a literatura, que confirmam os índices elevados do perfil epidemiológico de disfunções musculoesqueléticas.

\section{REFERÊNCIAS BIBLIOGRÁFICAS}

BRASIL. Resolução no 466 Conselho Nacional de Saúde. dezembro, 2012.

CERDEIRA ET AL. Fisioterapia no Sertão Central do Ceará: a caracterização dos pacientes atendidos em um ambulatório de reabilitação funcional. Revista Expressão Católica, v.2 n.1, p.24-40, 2013.

DAVID G. P. Perfil epidemiológico dos pacientes atendidos no setor de fisioterapia em ortopedia e traumatologia da clínica escola de fisioterapia da universidade São Francisco - análise retrospectiva. [Dissertação] Universidade São Francisco Curso De Fisioterapia Bragança Paulista, 2017.

FACULDADE SANTA MARIA (FSM). Policlínica. 2018. Disponível em: http://www.fsm.edu.br/sobre-nos/policlinica-da-faculdade-santa-maria. Acesso em: 25/11/2018.

JORGE A. F. AMBRÓSIO T. G. Perfil das afecções ortopédicas e traumatológicas dos pacientes de uma clínica de fisioterapia da cidade de Bragança Paulista - SP. [Dissertação] Universidade São Francisco Curso De Fisioterapia. Bragança Paulista 2016.

LEVORATO ET AL. Fatores associados à procura por serviços de saúde numa perspectiva relacional de gênero. Ciência \& Saúde Coletiva, v.19, n.4, p.1263-1274, 2014.

MORAES C.L.K. Perfil dos pacientes atendidos na Clínica Escola de Fisioterapia no setor de Ortopedia e Traumatologia. Revista Eletrônica Estácio Saúde, v.5, n.1, p.1617 - ISSN 1983, 2016.

NOGUEIRA, A.F. ET AL. Principais Distúrbios Traumato-Ortopédicos Atendidos Em ClínicasEscola De Fisioterapia. Revista Científica da Faculdade de Educação e Meio Ambiente, v.7, n.2 p.33-44, 2016. OLIVEIRA J.C. ET AL. Perfil Epidemiológico dos pacientes atendidos em uma clínica-escola de fisioterapia na cidade de Maceió-AL. Interfaces Científica, v.6, n.2, p.85, 2018.

OLIVEIRA, A.C; BRAGA, D.L.C. Perfil Epidemiológico dos pacientes na clínica de ortopedia da Universidade Paulista. J Health Sci Inst., Jundiaí, v.28, n.4, p.356-358, 2010.

PEREIRA ET AL. Perfil das internações de crianças e adolescentes com fraturas do crânio e ossos da face na região nordeste Brasil. Revista Brasileira de Ciências da Saúde, ISSN 14152177 v. 17, n.3, p.275-280, 2013.

RIOS, J.L.L., MARANHÃO, R.C., GAZINEU R.M.D., NOGUEIRA A.B., MARQUES C.D.L. Prevalência de queixas musculoesqueléticas em pacientes atendidos em ambulatório de ensino de Clinica Médica, Rev Bras Clin Med,v.7, p.283-289, 2009.

SACON, A. B.; PILLATT, A.P.; BERBAM, L.; FENGLER, V.Z.; BIGOLIN, S. E. Perfil de sujeitos atendidos na Clínica-escola de fisioterapia na área De ortopedia e traumatologia. Revista 
Contexto e Saúde, v. 10, n. 20. Rio grande do sul, 2011.

SANTOS, ET AL. Estudo epidemiológico do trauma ortopédico em um serviço público de emergência. Cad Saúde Colet., v.24 n.4 p. 97-403, 2016.

SILVA, K.O.C et. al. Perfil dos pacientes atendidos na clínica escola de fisioterapia no setor de ortopedia e traumatologia, Revista Eletrônica Estácio Saúde, v.4, n.1, p.53-58, Rio Grande do Norte, 2015.

SOUSA, A.R.B; RIBEIRO K.S.Q.S. A Rede Assistencial em Fisioterapia no Município de João Pessoa: uma Análise a Partir das Demandas da Atenção Básica. Revista Brasileira de Ciências da Saúde, v. 15, n. 3, p. 357-368, 2011.

WATANABE, L.A. Perfil epidemiológico dos pacientes atendidos no setor de fisioterapia de uma clínica de ortopedia em Goiânia. [Especialização]. Goiânia (GO): Universidade São Marcos; 2012. 